\title{
THE SIXTEENTH SUMMER MEETING OF THE AMERICAN MATHEMATICAL SOCIETY.
}

THE sixteenth summer meeting and sixth colloquium of the Society were held at Princeton University during the week September 13-18, 1909. The attendance included the following forty-one members :

Professor G. D. Birkhoff, Professor G. A. Bliss, Mr. R. D. Carmichael, Dr. A. B. Chace, Dr. A. Cohen, Professor F. N. Cole, Dr. G. M. Conwell, Dr. L. S. Dederick, Professor L. P. Eisenhart, Professor T. C. Esty, Professor H. B. Fine, Dr. G. F. Gundelfinger, Professor Harris Hancock, Rev. A. S. Hawkesworth, Dr. A. M. Hiltebeitel, Dr. J. G. Hun, Dr. Frank Irwin, Professor Edward Kasner, Professor W. R. Longley, Professor J. H. Maclagan-Wedderburn, Mr. H. F. MacNeish, Professor E. H. Moore, Professor Frank Morley, Professor Richard Morris, Professor G. D. Olds, Professor W. F. Osgood, Mr. H. W. Reddick, Mr. W. J. Risley, Professor D. A. Rothrock, Professor I. J. Schwatt, Dr. Clara E. Smith, Professor P. F. Smith, Professor Virgil Snyder, Professor Elijah Swift, Professor J. H. Tanner, Mr. C. E. Van Orstrand, Professor E. B. Van Vleck, Professor Oswald Veblen, Professor H. S. White, Professor J. E. Wright, Professor J. W. A. Young.

An account of the colloquium, which opened on Wednesday morning, will appear later in the BuLLETIN. The four sessions of the summer meeting proper occupied the first two days of the week. At the opening session Professor Fine occupied the chair, which was taken at the later sessions by Professor Morley and Vice-Presidents Kasner and Van Vleck. The Council announced the election of the following persons to membership in the Society : Dr. L. S. Dederick, Princeton University ; Dr. G. E. Wahlin, University of Illinois ; Mr. E. E. Whitford, College of the City of New York, Eleven applications for membership were received.

The sum of five thousand francs was appropriated from the treasury of the Society in support of the publication of the works of Euler and in subscription for a copy of these works for the Society's library. A committee was appointed to prepare and report at the October meeting suitable resolutions on the death of Ex-President Simon Newcomb. The committee 
on nominations, consisting of Professors E. B. Van Vleck, Brown, and Kasner, appointed to nominate officers and other members of the Council will also report at the October meeting.

At the closing session resolutions thanking the University for its hospitality and the committee on arrangements for their services were adopted. On Tuesday the members were conducted through the buildings and grounds of the University, and Tuesday evening was marked by a reception at the house of Professor Fine.

The following papers were read at this meeting :

(1) Professor L. P. Eisenhart: "Congruences of the elliptic type."

(2) Mr. Dunham Jackson : "Resolution into involutory substitutions of the transformation of a bilinear form into itself."

(3) Dr. F. W. ReEd : "On singular points in the approximate development of the perturbative function."

(4) Professor VIrgIL SNYDER : "Surfaces invariant under infinite discontinuous birational groups defined by line congruences."

(5) Mr. Joseph Lipke : "Natural families of curves in a general curved space," preliminary communication.

(6) Rev. A. S. Hawkesworth: "A new theorem in conics."

(7) Mrs. Anna J. Pell: "Applications of biorthogonal systems to integral equations."

(8) Mr. G. C. Evans : "The integral equation of the second kind, of Volterra, with singular kernel," preliminary communication.

(9) Professor Edward Kasner: "Triply orthogonal systems of surfaces."

(10) Professor Edward Kasner: "Natural families and Thomson's theorem."

(11) Professor G. A. Mrller: "The groups which may be generated by two operators $s_{1}, s_{2}$ satisfying the equation $\left(s_{1} s_{2}\right)^{\alpha}=\left(s_{2} s_{1}\right)^{\beta}$, $\alpha$ and $\beta$ being relatively prime."

(12) Dr. F. R. SharPe : "Integral equations with variable limits, with an application to the problem of age distribution."

(13) Mr. R. D. CaRmichaEL: "Note on a new number theory function."

(14) Professor T. E. McKinney : "On a criterion for $\lambda$-developments in the theory of equivalence."

(15) Dr. G. G. Chambers : "Groups of isomorphisms of the abstract groups of order $p^{2} q . "$ 
(16) Professor W. R. Longley : "Note on some periodic orbits with more than one axis of symmetry."

(17) Professor W. H. Bussey : "Tables of Galois fields of order less than 1,000."

(18) Professor W. B. Ford: "On the relation between the sum formulas of Hölder and Cesàro."

(19) Professor Oswald VEBlen : "Products of pairs of involutoric projectivities."

(20) Dr. G. F. Gundelfinger : "On the geometry of line elements in the plane with reference to osculating vertical parabolas and circles."

(21) Professor P. F. Sмrтн : "Theorems in the geometry of surface elements in space."

(22) Professor R. G. D. Richardson and Mr. W. A. HurWITZ: "Note on determinants whose terms are certain integrals."

(23) Professor R. G. D. Richardson: "The Jacobi criterion in the calculus of variations and the oscillation of solutions of linear differential equations of the second order."

(24) Professor I. J. SchwatT: "Methods for the summation of infinite series."

(25) Professor A. B. CoBle : "Cubic space curves that meet the Hessian of a cubic surface in six pairs of corresponding points." bility."

(27) Mr. H. W. REDDICK : "Geometric properties of a system of tautochrones."

(28) Dr. W. B. Carver: "The poles of finite groups of fractional linear substitutions in the complex plane."

(29) Dr. L. S. DEDERICK : "The solution of the equation in two real variables at a point where both the partial derivatives vanish."

(30) Dr. H. T. Burgess : "On point-circle correlations in the plane."

(31) Professor H. B. Newson : "A general theory of linear groups."

(32) Mr. A. R. Schweitzer : "A formal extension of Bolzano's series."

Mr. Jackson's paper was communicated to the Society by Professor Bôcher, Dr. Reed's by Professor White, and Mr. Evans's by Professor Osgood. Dr. Burgess was introduced by Professor Osgood. The papers of Dr. Sharpe and Dr. Carver 
were presented by Professor Snyder. The papers of Mr. Jackson, Dr. Reed, Mr. Lipke, Mrs. Pell, Mr. Evans, Professor Kasner's second paper, and the papers of Professor Miller, Professor McKinney, Dr. Chambers, Professor Bussey, Professor Ford, Professor Richardson and Mr. Hurwitz, Professor Richardson, Professor Coble, Professor Newson, and Mr. Schweitzer were read by title.

Abstracts of the papers follow below. The abstracts are numbered to correspond to the titles in the list above.

1. Kummer in his study of rectilinear congruences made use of two quadratic differential forms, namely $E d u^{2}+2 F d u d v$ $+G d v^{2}, e d u^{2}+\left(f+f^{\prime}\right) d u d v+g d v^{2}$, of which the first is the square of the linear element of the spherical representation of the congruence. When the Jacobian of these two forms, written $A d u^{2}+2 B d u d v+C d v^{2}$, is equated to zero, the resulting differential equation defines the developable ruled surfaces of the congruence. This equation admits of real solutions in the case of normal congruences, congruences of Guichard, cyclic congruences, and congruences of tangents to a real surface. But there is a large variety of congruences for which the solutions of this equation are imaginary, and yet little or no study has been made of them. It is with these congruences of the elliptic type that Professor Eisenhart's paper deals. By means of a theorem of Cifarelli it is found that through each line of a congruence there pass two real ruled surfaces such that when they are parametric, the relations $A / E=C / G, B=0$ obtain; they are called the characteristic ruled surfaces of the congruence. These ruled surfaces are found to possess properties analogous to certain well-known properties of the developable surfaces of a congruence of the hyperbolic type.

For a congruence of the elliptic type the abscissas of the focal points, measured from the middle point, are conjugate pure imaginary quantities. If the latter be denoted by $\rho$ and $-\rho$, the locus of the points at the distances $i \rho$ and $-i \rho$ constitute a pair of real surfaces which are called the pseudo-focal surfaces. When these surfaces are associate to one another, the congruence is of the kind discussed by Lilienthal. A further study of these congruences is made in terms of parameters so chosen that $A=C, B=0$, and these results are considered in connection with the congruences formed by the joins of corresponding points on associate surfaces. The paper closes with a discussion 
of the congruences whose developables cut the middle surface in the unicursal curves on the latter.

2. In Mr. Jackson's paper it is first pointed out that by combining a theorem recently proved by E. B. Wilson with an old result of Frobenius one can at once infer that a necessary and sufficient condition that a linear transformation be resolvable into the product of two involutory transformations is that there exist a non-singular bilinear form which this transformation carries over into itself. A new proof of the theorem of Wilson just referred to is then given.

3. Poincaré has shown how the higher terms in the development of the perturbative function may be derived approximately without computing the intervening terms. Applications of the theory to particular cases, principally in coplanar orbits, have been made by Coculesco, Féraud, and Hamy. Dr. Reed's paper considers the general case of elliptic orbits with any mutual inclination. The method is in part dependent upon numerical assumptions, but by use of a criterion for the admissibility of those singular points upon which the approximations depend the application can be made to orbits of any elements whatever. The solution of an ideal Jupiter-asteroid problem of general type is given in detail.

4. If the equation of a quadric contains a rational parameter to some degree higher than the first, and the two systems of generators are rationally separable, each system $\Sigma, T$ will define a line congruence having the envelope of the family for focal surface $\phi$. Each quadric will touch the focal surface in the points of an elliptic space quartic $C_{4^{\circ}}$. If a generator of $\Sigma$ touches $\phi$ in $P_{1}, P_{2}$, the operation of interchanging $P_{1}, P_{2}$ is involutional. Through $P_{2}$ passes a tangent to $\phi$ belonging to $T$, touching $\phi$ again in $Q_{2}$. The operation $\Sigma \mathrm{T}$ is now of infinite order.

For special relations among the coefficients, one, two, or three linear factors can be removed from $\phi$. Professor Snyder established the following theorem :

Surfaces of order four or any number greater than five can be constructed which are invariant under an infinite discontinuous group of birational transformations.

All the congruences of order 2 except those of class 7 are shown to belong to this category. The method is applied to 
two other congruences and the fundamental points of the transformation are determined.

5. In a recent article in the Transactions, ${ }^{*}$ a complete geometric characterization of the families of curves defined as the extremals connected with variation problems of the form $\int F d s=$ minimum (where $F$ is any point function and $d s$ is the element of arc in the space considered) is given for euclidean spaces of two and three dimensions; this may be easily extended to all spaces of constant curvature. In the present preliminary paper, Mr. Lipke gives a complete geometric characterization of natural families of curves in a curved space of two dimensions (general surface). The $\infty^{2}$ curves of any natural family in such a space have the following properties: (A) The locus of the centers of geodesic curvature of the $\infty^{1}$ curves through a point is a straight line; $(B)$ among the $\infty^{1}$ geodesic circles (circles of constant geodesic curvature) which osculate the $\infty^{1}$ curves through a point, there exist two hyperosculating circles which are orthogonal. The natural families are merely a special case of a much broader class of curves characterized by property $(A)$. The latter class also includes families of isogonal trajectories, which may be associated with and are gotten from the natural families by a curvature transformation.

6. Mr. Hawkesworth's paper is in abstract as follows: If two or more triangles to which any conic is in common escribed have each a vertex upon the same axis, and if in each case a circle be described passing through the triangle's other two vertices and with its center upon the axis, then the resultant system of circles will have the conic's other axis for their common radical axis passing in common through the two foci, or focoids, as the case may be, lying upon it, while their "limiting points" upon the first and given axis and line of their centers will be, in turn, the conic's focoids or foci. With other corollaries, the very important one follows that if any triangle cut by any transversal be given, then there is an easily found unique conic that has the transversal for an axis and is circumscribed by the triangle.

7. By considering a given complete biorthogonal system in

* "Natural families of trajectories : conservative fields of force." By Edward Kasner, Trans. Amer. Math. Society, April, 1909. 
connection with some of Hilbert's methods Mrs. Pell obtains the following results: Either the no -homogeneous integral equation

$$
\phi(s)=f(s)+\int_{a}^{b} K(s, t) \psi(t) d t
$$

where $\phi(s)$ and $\psi(s)$ are to be determined as associated functions with respect to the given biorthogonal system, has a solution $[\phi(s), \psi(s)]$; or the homogeneous integral equation

$$
\phi(s)=\int_{a}^{b} K(s, t) \psi(t) d t
$$

has. If the unsymmetrical kernel $L(s, t)$ is the associated function of a symmetrical kernel there always exist characteristic values $\lambda_{i}$ and corresponding characteristic solutions

$$
\phi_{i}(s)=\lambda_{i} \int_{a}^{b} L(s, t) \phi_{i}(t) d t, \quad \psi_{i}(s)=\lambda_{i} \int_{a}^{b} L(t, s) \psi_{i}(t) d t .
$$

The solutions $\left[\phi_{i}(s), \psi_{i}(s)\right]$ form a biorthogonal system equivalent to the given one. Any function $f(s)$ expressible in the form

$$
f(s)=\int_{a}^{b} L(s, t) f_{1}(t) d t
$$

can be developed in a uniformly convergent series of the form

$$
f(s)=\sum_{i=1}^{\infty} \phi_{i}(s) \int_{a}^{b} \psi_{i}(s) f(s) d s .
$$

9. Professor Kasner obtains a set of general relations involving the curvatures of the three surfaces and the curvatures of of their curves of intersection. The more general theory of triply orthogonal congruences of curves is also studied. Corollaries are given for plane curves.

10. In Professor Kasner's second paper it is shown that the orthogonality properties involved in Thomson's theorem are sufficient to characterize natural families of trajectories. Stronger converse results are also obtained.

11. Professor Miller's paper appears in full in the present number of the BuLLETIN. 
12. The integral equation of the second kind with variable limits which have a constant difference has been solved by $\mathrm{P}$. Hertz and C. Herglotz (Mathematische Annalen, volume 65). In Dr. Sharpe's paper a more direct and shorter solution is obtained by considering the limit of a finite difference equation with constant coefficients. The complete solution is found for the case in which a certain transcendental equation has equal roots. An application is made to the problem of the age distribution of population. It is shown that the final distribution is ultimately independent of the initial distribution.

13. By means of Euler's $\phi$-function Mr. Carmichael defines $\lambda(n)$ as follows: $\lambda\left(p^{a}\right)=\phi\left(p^{a}\right)$ when $p$ is an odd prime; $\lambda\left(2^{a}\right)=\phi\left(2^{a}\right)$ when $a=1$ or $2 ; \lambda\left(2^{a}\right)=\frac{1}{2} \phi\left(2^{a}\right)$ when $a>2$; $\lambda\left(2^{a} p_{1}^{a_{1}} \cdots p_{i}^{a_{i}}\right)=$ the lowest common multiple of $\lambda\left(2^{a}\right)$, $\lambda\left(p_{1}^{a_{1}}\right), \cdots, \lambda\left(p_{i}^{a_{i}}\right), p_{1}, \cdots, p_{i}$ being different odd primes. If $\lambda(n)$ is the exponent to which $a$ belongs modulo $n$, then $a$ is called a primitive $\lambda$-root of $x^{\lambda(n)}=1(\bmod n)$. The following are some of the theorems obtained:

For any given $n$ the congruence $x^{\lambda(n)} \equiv 1(\bmod n)$ is satisfied by every $x$ prime to $n$. In every congruence $x^{\lambda(n)} \equiv 1(\bmod n)$ a solution $g$ exists which is a primitive $\lambda$-root, and for any such $g$ there are $\phi\{\lambda(n)\}$ primitive $\lambda$-roots congruent to powers of $g$. If $\lambda(n)>2$ the product of primitive $\lambda$-roots congruent to powers of $g$ is congruent to $1(\bmod n)$. If $x_{1}$ is the largest value of $x$ satisfying the equation $\lambda(x)=a$, any other solution $x_{2}$ is a factor of $x_{1}$. Let $\alpha$ be that divisor of $\alpha$ for which $\lambda(x)=a$ has a greatest solution $x_{1}$ greater than such a solution when for $\alpha$ any other divisor of $\alpha$ is taken. Then $x_{1}$ is the largest divisor of $z^{a}-1$ for every $\approx$ prime to the divisor. Finally it is shown that there are values of composite $n$ for which the relation $e^{n-1} \equiv 1(\bmod n)$ is true when $e$ is any number prime to $n$, and a method is given for finding such values of $n$.

14. In a paper "Concerning a certain type of continued fractions depending on a variable parameter" in the American Journal of Mathematics, volume 29, number 3, Professor McKinney showed that when $X_{0}$ is properly equivalent to a "critical value" $x_{0}$, then $\lambda\left(X_{0}\right)_{\lambda^{\prime}}$ may be ultimately like $\lambda\left(x_{0}\right)_{\lambda^{\prime}}$ or ultimately like the "associate" of $\lambda\left(x_{0}\right)_{\lambda^{\prime}}$. In that paper no criterion was established for deciding between these two cases. It is the object of the present paper to set up a criterion for an important class of critical values. 
15. The paper by Dr. Chambers takes up the non-abelian abstract groups of order $p^{2} q$ as determined by Hölder, and finds for each its corresponding group of isomorphisms and the group of cogredient isomorphisms. Independent generators for these groups of isomorphisms are obtained in terms of the generators of the original groups, and sufficient relations among these generators are determined to define the groups of isomorphisms.

16. The discussion of the periodic orbits of a particle which is subject to the newtonian attraction of finite bodies whose motion is supposed to be known, begins naturally with Jacobi's restricted problem of three bodies. In this case all the periodic orbits of the particle in the plane of motion of the finite bodies have one axis of symmetry, namely, the line joining the finite bodies. The symmetrical properties are important, both in the numerical processes of Darwin * and the analysis employed by Moulton. $f$ In generalizing the problem by introducing a greater number of finite bodies, the next step is to consider three spheres moving in circles according to the equilateral triangle solution of Lagrange. This problem has been treated in detail. $\$$ The periodic orbits of the particle have no axis of symmetry, or they have one such axis, depending upon the distribution of mass. Professor Longley's note considers certain cases in which the particle is subject to the attraction of more than three finite bodies revolving in circles about their center of mass. Some of the periodic orbits of the particle about one of the finite bodies have more than one axis of symmetry, and, as a consequence, it is possible to make some simplifications in the analysis used for the preceding cases.

17. In the Bulletis, volume 12 (1905), pages 21-38, Professor Bussey published a paper entitled "Galois field tables for $p^{n} \leqq 169$." The present paper is an extension of that work and contains tables of all Galois fields of order $p^{n}$ such that $n>1$ and $169<p^{n}<1,000$. The limit 1,000 is that set by Jacobi in his Canon Arithmeticus, which contains tables of indices for all primes less than 1,000. These tables of indices are tables of all Galois fields of prime order. When this paper is published, there will be in print tables of all Galois fields of order less than 1,000 .

* Acta Mathematica, vol. 21 (1897), p. 99.

† Transactions Amer. Math. Society, vol. 7 (1906), p. 537.

† Ibid., vol. 8 (1907), p. 159. 
18. Professor Ford's paper is devoted to a proof of the folfowing theorem : "If a given series (convergent or divergent) is summable and of indeterminacy $r$ in Hölder's sense, it is summable and of indeterminacy $r$ in Cesàro's sense, and conversely." The direct part of this theorem has already been established by Knopp in his dissertation "Grenzwerte von Reihen bei der Annäherung an die Konvergenzgrenze" (Berlin, 1907), while the converse has been proved for the special cases in which $r=1,2$ by Bromwich (Mathematische Annalen, volume 65 (1908), pages 363-365). The above theorem supplements these investigations by establishing both the direct and converse theorems for all values of $r$. It follows that the sum formulas of Hölder and Cesàro are coextensive in their applicability to given series and determine one and the same sum.

The paper has been offered to the American Journal of Mathematics.

19. Professor Veblen's paper has to do with a class of theorems of which Pascal's theorem on conic sections is one and of which another is the following: If a simple hexagon is inscribed in a surface of the second degree, each pair of opposite sides is met by a pair of lines conjugate to both sides, and the latter three pairs of lines are met by one pair of lines.

20. The investigations made by Dr. Gundelfinger in this paper arise from an interpretation in the plane of the geometry within a linear line complex by means of a transformation due to Sophus Lie.* This transformation takes a point of space into a line element of the plane; curves of the complex into unions; complex lines, in particular, into vertical parabolas ; - hence the tangents to a complex curve transform into the vertical parabolas which osculate the corresponding union.

From a classification of curves and of surfaces with respect to the group $G_{10}$ of projective transformations in space which leave the linear line complex invariant we obtain a classification of " line element loci" of $\infty^{1}$ and of $\infty^{2}$ elements with respect to the $\Gamma_{10}$ of contact transformations in the plane - that is we classify ordinary differential equations of the first order with respect to the contact relations of the $\infty^{2}$ vertical parabolas which osculate their integral curves.

The fact that the linear line complex is invariant also under the correlation which it defines when regarded as a null system 
gives rise to a theory of " reciprocal line element loci" of much interest. Furthermore, the various types of congruences of complex lines, characterized by the nature of their focal surface the two sheets of which are reciprocal configurations, give rise to all possible arrangements of $\infty^{2}$ vertical parabolas in the plane which are distinct in regard to the singular solution of the corresponding differential equation of the second order.

By using a second transformation of Lie's $\dagger$ which differs from the above in that complex lines go over into circles instead of vertical parabolas, a second interpretation is effected giving rise to many interesting theorems on the osculating circle.

21. In recent investigations of Scheffers and Lilienthal some interesting theorems are demonstrated on the osculating circles of the integral curves of an ordinary differential equation of the first order. Professor Smith's paper recasts these results in such a way that generalization to partial differential equations is made possible. It is found that the rôle played by the osculating circles is assumed in space by osculating vertical parabolic bands, that is, bands on the surface of a parabolic cylinder between any two parallel vertical planes indefinitely near. It is shown that the osculating vertical parabolic bands of the characteristic bands of an arbitrary partial differential equation of the first order belong to a second partial differential equation - the reciprocal of the former. Exceptional cases are easily characterized. The results follow readily from the projective geometry within a null-system in five-dimensional space, a subject which has recently received the attention of Professor Eiesland.

22. The paper by Professor Richardson and Mr. Hurwitz appeared in full in the October number of the Bulletin.

23. Hilbert has recently discovered $*$ the connection between the calculus of variations and the theories of differential and integral equations and has emphasized the fact that the former theory is the wider in its scope. The self-adjoint differential equation of the second order $\left(p u^{\prime}\right)^{\prime}+q u+\lambda k u=0$ containing the parameter $\lambda$ may be regarded as the Lagrange equation of a calculus of variation problem with a certain quadratic subcondition or with this quadratic and certain linear sub-conditions. The questions concerning the necessary and sufficient

* Geometrie der Berührungstransformationen, p. 238.

† Ibid., p. 245. 
conditions for the existence of a minimum, in particular the significance of the Jacobi criterion are discussed in this paper by Professor Richardson. The chief theorem may be stated as follows: The Jacobi criterion demands that the solution $u(x)=U_{1}(x)$ of the problem

$\int_{0}^{1}\left(p u^{\prime 2}-q u^{2}\right) d x=\min ,[p(x)>0, q(x) \leqq 0, u(0)=u(1)=0]$,

with the quadratic sub-condition

$$
\int_{0}^{1} k(x) u^{2} d x=1
$$

shall not vanish in the interval $(0,1)$, that the solution $u(x)=U_{2}(x)$ of the same problem with the quadratic and one linear sub-condition

$$
\int_{0}^{1} k U_{1}(x) u(x) d x=0
$$

shall vanish once in the interval, and in general that the solution $u(x)=U_{n+1}(x)$ of the problem with the quadratic and $n$ linear sub-conditions

$$
\int_{0}^{1} k(x) U_{1}(x) u(x) d x=0, \ldots, \int_{0}^{1} k(x) U_{n}(x) u(x) d x=0
$$

shall vanish exactly $n$ times in the interval.

This paper is the first of a series to appear in the Mathematische Annalen.

24. Professor Schwatt developed certain methods for the summation of special form of infinite series.

25. Professor Coble's paper deals with two kinds of cubic space curves associated with a general cubic surface.

The curve of the one kind has six skew lines of a double six of the surface as axes. It contains $\infty^{2}$ sets of five osculating planes whose ten meets are on the surface. There are 72 systems of these curves, each system containing $\infty^{2}$ curves.

The curve of the other kind meets the Hessian of the surface in six pairs of corresponding points. It contains $\infty^{2}$ sets of five

\footnotetext{
* "Grundzüge einer allgemeinen Theorie der linearen Integralgleichungen"; Göttinger Nachrichten, 1 und 2 Mittheilungen 1904, 4 und 5 Mittheilungen 1906.
} 
points any three of which are an apolar triad of the surface. There are 16 systems of these curves, each system containing the $\infty^{2}$ curves on a set of five points found among the poles of the planes of the Sylvester pentahedron.

26. A solution $\left(x_{1}, x_{2}, \cdots, x_{n}\right)$ of the system of ordinary differential equations

$$
\frac{d x_{i}}{d t}=X_{i}\left(x_{1}, x_{2}, \cdots, x_{n}\right) \quad(i=1,2, \cdots, n)
$$

is defined to be stable if $x_{1}, x_{2}, \ldots, x_{n}$ remain finite for $t>t_{0}$. This paper by Professor Birkhoff is a study of such solutions, especially with reference to their periodic or quasi-periodic character.

27. The totality of tautochrones in a plane, due to a force whose rectangular components are functions only of the coordinates of the point at which the force acts, forms a system of $\infty^{3}$ curves. In the July number of the Bulletin Professor Kasner gave the intrinsic equation of such a system. In the present paper Mr. Reddick deduces the cartesian equation of the system, a differential equation of the third order, and investigates its geometric properties. The necessary and sufficient conditions that any plane system of $\infty^{3}$ curves shall be a family of tautochrones are obtained.

28. In his Lectures on the icosahedron, Klein shows that any finite group of fractional linear substitutions on a complex variable can be reduced to a canonical form such that each substitution will correspond (by the ordinary stereographic projection) to a rotation of the sphere which has the unit circle of the complex plane as a great circle. The poles of each substitution are what we may call "skew-inverse" points with respect to the unit circle.

The following questions, which are in fact equivalent, naturally arise :

(1) If a finite group of fractional linear substitutions is not in this canonical form, do its operations correspond in the same way to rotations of a sphere having some other circle of the complex plane as a great circle?

(2) If upon all pairs of skew-inverse points with respect to a circle in the complex plane, one operates with an arbitrary fractional linear substitution, will they go into skew-inverse points with respect to some circle; and if so, what circle? 
In the present paper Dr. Carver answers the second question; and hence also the first.

29. In an earlier paper, ${ }^{*}$ Dr. Dederick gave sufficient conditions for the existence of a solution of the equation $F(x, y)=0$, in the form $y=f(x)$ in the neighborhood of a point where $\partial F / \partial x=\partial F / \partial y=0$, and for the existence and continuity of $d y / d x$ in the case where the equation for formally determining $d y / d x$ has a simple root. The present paper extends these results to the higher derivatives of $y$ with regard to $x$, and to the case of a multiple root of the equation for $d y / d x$. The results may be summed up in the statement: The ordinary process for determining the character of the branches of the plane curve $F(x, y)=0$ at a singularity, and the method of differentiating $F(x, y)$ totally with regard to $x$ or with regard to a parameter $t$, in order to obtain the successive derivatives of $y$ with regard to $x$ or of $x$ and $y$ with regard to $t$, are justified if the partial derivatives of $F(x, y)$ which are used are continuous at and near the point in question.

30. The problem of the determination and discussion of point-circle correlations in the plane from the standpoint of the geometry of contact transformations is treated in Dr. Burgess's paper. The method used involves those questions in the geometry on a quadric which arise in point-plane correlations in space. Algebraic difficulties make it necessary to limit the scope of the paper to a complete solution of the involutory cases - a solution effected by elementary divisors. The Cayley numbers for a curve on a quadric are interpreted for its stereographic projection, and formulas given by which the Cayley numbers for the transformed curve may be found.

The æquatio-directrix of the transformation suggests two problems: (1) the study of the properties of a single transformation, and (2) the study of a system of transformations in one parameter. In certain cases, this system turns out to be a group.

31. A general theory of linear groups which applies equally to continuous and discontinuous groups in any number of variables was announced in Professor Newson's paper. If no restrictions are laid upon the variation of the elements of the matrix $M$ of a linear transformation $T$ in $n$ variables, the continuous group $G_{n^{2}-1}$ of $\infty^{n^{2}-1}$ collineations in space of $n-1$

\footnotetext{
* Presented at the April meeting of the Society.
} 
dimensions is obtained. A subgroup of $G_{n^{2-1}}$ is obtained when the elements of $M$ satisfy certain conditions, as $e . g$., the wellknown conditions defining the orthogonal group. Professor Newson's fundamental theorem lays down the necessary and sufficient conditions which its elements of $M$ must satisfy in order to have a subgroup of $G_{n^{2}-1}$. He defines a complete family of automorphic forms $\phi_{i}$ which are homogeneous and symmetric functions in from 1 to $n$ sets of $n$ variables each. His theorem is: A necessary and sufficient condition for the existence of a subgroup of $G_{n^{2}-1}$ is that the elements of $M$ satisfy a set of equations $\phi_{i}=l_{i}$ consisting of a complete family of automorphic forms in the elements of the rows or columns of $M$, each equated to the corresponding coefficient of the family.

Families of lower degrees define continuous subgroups of $G_{n^{2-1}}$; after a certain degree is reached the subgroups become discontinuous; above a certain other degree the conditions are satisfied only by the identical transformation. The paper will be published in the Kansas University Science Bulletin.

32. Mr. Schweitzer contrasted the formal properties of Bolzano's linear series with his exposition of the series of Vailati (the system ${ }^{1} R_{1}$ ) and showed how to extend Bolzano's series to $n$ dimensions $(n=1,2,3, \ldots)$ by considering simple modifications of the axioms of dimensionality and extension in his system ${ }^{n} R_{n}$. Application of the author's $n$-dimensional open and closed chains is made.

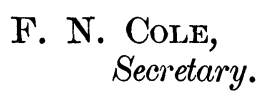

\section{THE GROUPS WHICH MAY BE GENERATED \\ BY TWO OPERATORS $s_{1}, s_{2}$ SATISFYING \\ THE EQUATION $\left(s_{1} s_{2}\right)^{a}=\left(s_{2} s_{1}\right)^{\beta}, \alpha$ AND $\beta$ BEING RELATIVELY PRIME.}

BY PROFESSOR G. A. MILLER.

(Read before the American Mathematical Society, September 13, 1909.)

Since $s_{1} s_{2}$ and $s_{2} s_{1}$ are of the same order and $\alpha, \beta$ are relatively prime, it results that this common order is prime to both $\alpha$ and $B$. Hence $s_{1} s_{2}$ and $s_{2} s_{1}$ are generated by either $\left(s_{1} s_{2}\right)^{\alpha}$ or $\left(s_{2} s_{1}\right)^{\beta}$, and the cyclic group generated by $s_{1} s_{2}$ coincides with the one generated by $s_{2} s_{1}$. A direct consequence of this is that the group generated by $s_{1} s_{2}$ is invariant under the entire group $G$ 